\title{
Transient elastic waves propagating in a multi-layered medium subjected to in-plane dynamic loadings II. Numerical calculation and experimental measurement
}

\author{
By C.-C. M A A D G.-S. LEE \\ Department of Mechanical Engineering, National Taiwan University \\ Taipei, Taiwan 10617, Republic of China
}

Received 11 February 1999; revised 5 August 1999; accepted 20 September 1999

The transient responses in the time domain of a layered medium subjected to dynamic in-plane loadings are presented in this part. Cagniard's method will be employed to invert the matrix series solution in the transformed domain (which was obtained in the first part of the paper) to the time domain. The numerical results for the transient response of a thin layer overlaying a half-space is presented and is discussed in detail. Acoustic emission experiments were conducted to study the propagation of elastic waves in the aforementioned medium, and to verify the numerical results. The experimental results show that the early-time transient response of vertical displacements on the surface of the thin layer agrees very well with the theoretical solutions under the plane stress assumption.

Keywords: transient response; two-layered medium; surface displacement

\section{Introduction}

By the application of the matrix Bromwich expansion method, the transient wave solution in a two-dimensional elastic strip was derived in the work of authors Ma \& Lee (1999). Good agreement between the numerical calculations and experimental measurements was obtained. The method of analysing theoretical solutions for a strip is extended to construct the exact solution for the two-dimensional multi-layered medium in the first part of this paper. The second part mainly concerns the numerical calculations and experimental measurements on a layered half-space.

As a degenerated case, the transient responses of a thick plate were studied in great detail by Pao and his co-workers (Pao \& Gajewski 1977; Pao 1978; Ceranoglu \& Pao 1981) through the generalized ray method. The transient waves generated by a buried compressional pulse in a two-layered solid (a layer overlaying a half-space) were calculated theoretically and numerically by Abramovoci \& Alterman (1965) and Alterman \& Karal (1968), respectively. This work was based on the numerical evaluation of the exact solution and the analysis was extremely involved and time consuming. Müller $(1968 a, b, 1969)$ used the method of generalized ray to determine the transient responses for a multi-layered medium in principle and calculated 

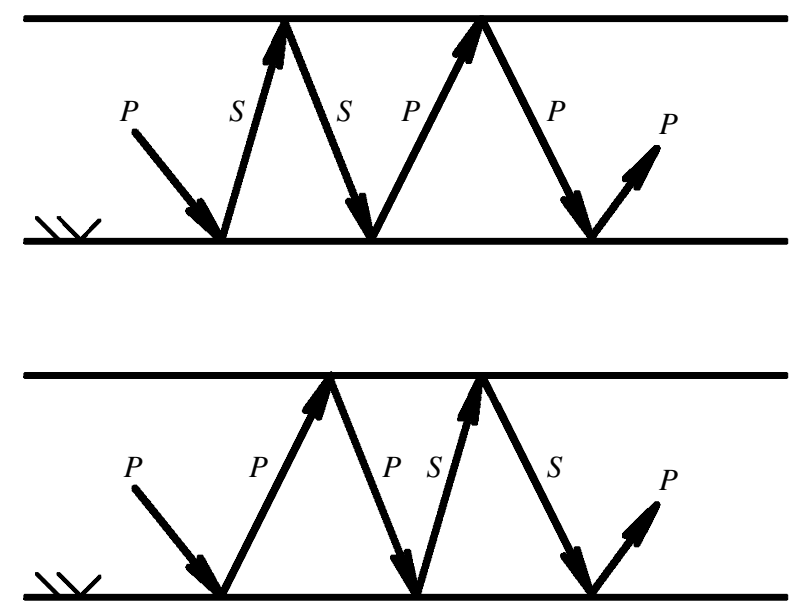

Figure 1. An example of degeneration of waves.

some reflected and head waves in three models of the Earth's crust. However, simple computational formulae are not available to obtain the total wave field within the multi-layered medium. In this paper, a formula including all waves propagating in the multi-layered medium is used to calculate a complete exact transient response. Such an exact solution would be of use from a practical point of view, either for checking the various approximations or for the understanding of some phenomena which are not easily describable by the use of any approximations. The formulae presented in this study enable us to calculate the long-time transient responses for the layered medium.

The determination of the motion on the free surface and in the interior of a layered elastic solid due to an impulsive source has received a considerable attention in the application of non-destructive evaluation of materials. Acoustic emission (AE) is the spontaneous release of transient elastic waves caused by sudden localized changes in stress within a body. The elastic waves generated by crack growth or deformation are frequently detected by an array of transducers attached to the free surface of the sample or structure. Analysis of measured elastic waveforms can, in principle, be used to characterize defect severity. The underlying principles and basic theories for the source location, source mechanism, signal dispersion and source characterization of acoustic emission can be found in an article of Pao (1978).

Sherwood (1958) calculated the transient responses of a semi-infinite solid subjected to a line loading, and compared theoretical results with experimental measurements. Breckenridge et al. (1975) showed that the theoretical and experimental waveforms can agree very well by using the breaking glass capillaries on a large block of aluminium to simulate the impulsive vertical force with unit step-time dependence acting on a half-space. The experimental waveform generated by breakage of capillaries and measured on a thick plate was compared with the theoretical calculation by Hsu et al. (1977) and Proctor et al. (1983). Ma \& Lee (1999) showed the good agreement between theoretical calculations and experimental waveforms on a strip subjected to an in-plane loading. In this paper, in order to verify the numerical calculations, the experiments were conducted on a layer of strip overlying a halfspace. 
The paper is organized as follows. Section 2 describes the method to construct the transient response for the layered medium by inverting the transformed matrix solution through Cagniard's method. The complete transient solution in the time domain including all waves propagating in the layered medium is presented. Section 3 gives the formal solution for a layer of strip overlying a half-space and discusses further, for the special case of a layered medium, the meaning of the reflection and transmission matrix $\boldsymbol{R}$ given in the first part of this paper. In $\S 4$, the numerical results obtained by using the exact transient solution are discussed. It is found that Stoneley-like waves will occur in the numerical calculation even when the material parameters of the problem lie outside the region for which they are predicted. This is in agreement with the findings of Gilbert \& Laster (1962). The surface motions of a layer overlying a half-space were measured experimentally, and compared with the theoretical calculation. The good agreements between experimental and theoretical waveforms imply the accuracy of numerical calculation and the possible application of the result to the detection of a perfect bonding condition.

\section{The transient response in time domain for the layered medium}

The complete double Laplace transformed solution in terms of matrix series for a layered medium subjected to in-plane dynamic loading was given in part I. The method of constructing the transient response in time domain is described in detail in this section.

The matrix formulation given in eqn (6.1) of part I is recognized as a superposition of infinite groups of waves that are reflected by, or transmitted through, the interface at the same times. The waves in each group are collected automatically in the matrix formulation and represented by double Laplace transformed potentials which can be inverted into time domain using the method proposed by Cagniard (1939).

The ray-tracing technique, which is a coding method used for analysing the transient waves in a strip, was developed by Ma \& Lee (1999) to distinguish each ray in the matrix formulation. Different types of waves in the theory of generalized ray can have the same expression of potential and this phenomenon is noted as degeneration of waves. For example, the case of degeneration arises when the waves are reflected five times by the free traction boundary of a strip, as shown in figure 1 . When we attempt to take account of all the waves which are multiply reflected within the strip, the problem of wave degeneration will be encountered immediately. Because the degenerate rays are identical, only the composite events have physical significance. Furthermore, the degenerate events increase significantly as the number of reflections increases. A better way must be sought for removing the degeneration, otherwise the ray-tracing technique becomes extremely inefficient from the computational standpoint.

For waves propagating in the multi-layered medium, the problem of degeneration can be obviated by collecting the terms with the same coefficients in the power matrix series of the phase-related reflection and transmission matrix $\boldsymbol{R}$. Since the reflected and transmitted waves are induced by the interfaces in successive order, the recursive formulation can be used to obtain the reflections or transmissions of $i$ th order from those of the $(i-1)$ th order. That is, the $n$th power of $\boldsymbol{R}$ for the $i$ th reflection or transmission can be obtained in a recursive sense,

$$
\boldsymbol{R}^{i}=\boldsymbol{R} \boldsymbol{R}^{i-1}, \quad i=1,2, \ldots, \infty .
$$

Proc. R. Soc. Lond. A (2000) 
The terms with like coefficients are collected during the process of matrix multiplication. Since the degeneration is dependent merely on the phase-related reflection and transmission matrix $\boldsymbol{R}$, the power matrix of $\boldsymbol{R}$ can be worked out in a symbolic form beforehand by a FORTRAN program, to facilitate the numerical calculation.

Consequently, the matrix solution in eqn (6.1) of part I can be rewritten in its component form as follows

$$
\hat{b}_{l}=\sum_{q=1}^{4 n}\left(R_{c v}\right)_{l q} s_{0 q}^{*}+\sum_{i=1}^{\infty} \sum_{r=1}^{4 n} \sum_{q=1}^{4 n}\left(R_{c v}\right)_{l r}\left(R^{i}\right)_{r q} s_{q}^{*},
$$

where the subscript $l$ varies from 1 to $4 n$ and the symbol $\left(R^{i}\right)_{r q}$ denotes the $(r-q)$ entry of power matrix $\boldsymbol{R}^{i}$. The matrix solution reduces to a canonical form with the application of Cagniard's method by combining the phase factors in the source vector $s^{*}$ with those in receiver matrices $\boldsymbol{R}_{\boldsymbol{c} v}$. The closed-form solution can be expressed as

$$
\begin{aligned}
\hat{b}_{l}=F_{l}(p) \sum_{q=1}^{4 n}\left(R_{c v}^{\prime}\right)_{l q} s_{0 q}^{\prime *} \exp \left[-p \sum_{k=1}^{n}\left(y_{\mathrm{L}}^{(k)} \gamma_{\mathrm{L}}^{(k)}+y_{\mathrm{T}}^{(k)} \gamma_{\mathrm{T}}^{(k)}\right)\right] \\
+F_{l}(p) \sum_{i=1}^{\infty} \sum_{j=1}^{m} \sum_{r=1}^{4 n} \sum_{q=1}^{4 n} d_{i j r q}\left(R_{c v}^{\prime}\right)_{l r} \Pi_{i j r q} s_{q}^{*} \exp \left[-p \sum_{k=1}^{n}\left(y_{\mathrm{L}}^{(k)} \gamma_{\mathrm{L}}^{(k)}+y_{\mathrm{T}}^{(k)} \gamma_{\mathrm{T}}^{(k)}\right)\right]
\end{aligned}
$$

where $\left(R_{c v}^{\prime}\right)_{l r}$ and $s_{q}^{\prime *}$ are the functions whose factors merely depend on the Laplace transform parameter $\xi$ in $\boldsymbol{R}_{\boldsymbol{c} v}$ and $\boldsymbol{s}^{*}$, respectively. The submultiple $F_{l}(p)$ depends only on the transform parameter $p$, which is factored from $\boldsymbol{R}_{\boldsymbol{c} v}$ and $\boldsymbol{s}^{*}$. In (2.3), the entries of power matrix $\boldsymbol{R}^{i}$ are expressed in terms of a finite series of products of the reflection or transmission coefficients, as well as the phase factor. Removing the degeneration of the multiply reflected waves, $m$ distinguishable waves, each of which has $d_{i j r q}$ duplicates in the $(r-q)$ entry of $\boldsymbol{R}^{i}$, can be obtained. The function $\Pi_{i j r q}$, which merely depends on $\xi$, is the product of the reflection or transmission coefficients without phase factors in $\boldsymbol{R}^{i}$. In the expression of the phase factor, $y_{\mathrm{L}}^{(k)}$ and $y_{\mathrm{T}}^{(k)}$ are determined by collecting the corresponding phase factors in $\boldsymbol{R}_{\boldsymbol{c} v}, \boldsymbol{R}$ and $\boldsymbol{s}^{*}$. Taking into account generalized ray theory, $y_{\mathrm{L}}^{(k)}$ and $y_{\mathrm{T}}^{(k)}$ are the projections along the $y$-axis of the ray path in $P$ and $S$ mode of the $k$ th layer, respectively.

The canonical form of each term in (2.3) can be written as follows

$$
\hat{E}=F(p) G(\xi) \exp \left[-p \sum_{k=1}^{n}\left(y_{\mathrm{L}}^{(k)} \gamma_{\mathrm{L}}^{(k)}+y_{\mathrm{T}}^{(k)} \gamma_{\mathrm{T}}^{(k)}\right)\right],
$$

where $G(\xi)$ is a function of $\xi$, involving the source function, receiver function and the product of the reflection and transmission coefficients. To perform Cagniard's method, the inversion of the Laplace transform parameter $\xi$ for this canonical expression is rewritten formally as follows:

$$
\bar{E}(p)=\frac{p F(p)}{2 \pi \mathrm{i}} \int_{\xi_{1}-\mathrm{i} \infty}^{\xi_{1}+\mathrm{i} \infty} G(\xi) \exp \left\{-p\left[\sum_{k=1}^{n}\left(y_{\mathrm{L}}^{(k)} \gamma_{\mathrm{L}}^{(k)}+y_{\mathrm{T}}^{(k)} \gamma_{\mathrm{T}}^{(k)}\right)-\xi x\right]\right\} \mathrm{d} \xi .
$$


The bar is used to denote the transform on time $t$. Cagniard's method deals with the inverse Laplace transform of the integral in (2.5). Once the inverse Laplace transform of the integral in (2.5) is obtained, the final solution corresponding to the canonical term in (2.3) can be calculated through a convolution integral.

The idea of Cagniard's method is to deform the path of integration in the complex $\xi$-plane along a new path in such a manner that the inverse Laplace transform of the integral along the new path of integration can be obtained by inspection. The application of Cagniard's method was explained in detail in the textbook by Achenbach (1973), and the result is stated as follows

$$
E(t)=\frac{1}{\pi} \mathrm{L}^{-1}\{p F(p)\} * \operatorname{Im}\left\{G(\xi) \frac{\partial \xi}{\partial t} H\left(t-t_{\mathrm{a}}\right)\right\}_{\xi=\xi_{1}},
$$

where $H(\cdot)$ is the unit step-time function, $\mathrm{L}^{-1}\{\cdot\}$ denotes the inverse Laplace transform in $t$ and $*$ represents the convolution of two functions with respect to time $t$. In this expression, $\xi_{1}$ is the root given by the following equation, which can be evaluated numerically by the modified Newton's method:

$$
t=\sum_{k=1}^{n}\left(y_{\mathrm{L}}^{(k)} \gamma_{\mathrm{L}}^{(k)}+y_{\mathrm{T}}^{(k)} \gamma_{\mathrm{T}}^{(k)}\right)-\xi x .
$$

The arrival time of each distinct wave, denoted as $t_{\mathrm{a}}$, is determined from the stationary value of $t$, which is a function of $\xi_{1}$, providing that the Cagniard path does not pass through the branch cut on complex $\xi$-plane. The global stationary value for $t$ is given by the condition

$$
\left(\frac{\partial t}{\partial \xi_{1}}\right)_{\xi_{1}=\xi_{\mathrm{M}}}=0=-\sum_{k=1}^{n}\left(\frac{y_{\mathrm{L}}^{(k)} \xi_{\mathrm{M}}}{\sqrt{s_{\mathrm{L}(k)}^{2}-\xi_{\mathrm{M}}^{2}}}+\frac{y_{\mathrm{T}}^{(k)} \xi_{\mathrm{M}}}{\sqrt{s_{\mathrm{T}(k)}^{2}-\xi_{\mathrm{M}}^{2}}}\right)-x .
$$

It can be shown that the foregoing equation has only one real root, which is located in the interval $\left[-s_{\min }, 0\right]$ for $x<0$ or $\left[0, s_{\min }\right]$ for $x>0$, where $s_{\text {min }}$ is the minimal slowness which appeared in (2.7). Equations (2.7) and (2.8) give the arrival time of the wave. If the Cagniard path encloses the branch point, a head wave that arrives earlier than the body wave is generated. The arrival time of this wave, $t_{\mathrm{a}}$, will be given by substituting $\xi$ in $(2.7)$ with $-s_{(i)}$, the value of the branch point which is enclosed by the Cagniard path. Note that (2.7) and (2.8) define a closed curve in space, which varies with $t$; the curve is called the 'wave front'.

Since the convolution integral in (2.6) has singularities at the second part when $\partial t / \partial \xi$ vanishes at the wave front, the convolution should be carried out carefully. The singularities can be reduced by the following formulation

$$
\begin{aligned}
E(t) & =\frac{1}{\pi} \mathrm{L}^{-1}\left\{p^{2} F(p)\right\} * \int_{0}^{t} \operatorname{Im}\left\{G(\xi) \frac{\partial \xi}{\partial \tau} H\left(\tau-\tau_{\mathrm{a}}\right)\right\}_{\xi=\xi_{1}(\tau)} \mathrm{d} \tau \\
& =\frac{1}{\pi} \mathrm{L}^{-1}\left\{p^{2} F(p)\right\} * H\left(t-t_{\mathrm{a}}\right) \operatorname{Im} \int_{0}^{\xi_{1}(t)} G(\xi) \mathrm{d} \xi,
\end{aligned}
$$

where we assume that $f(0)=f^{\prime}(0)=0$, in which $f(t)$ is the inverse transform of $F(p)$. The integral in (2.9) is then calculated on the complex $\xi$-plane by contour 


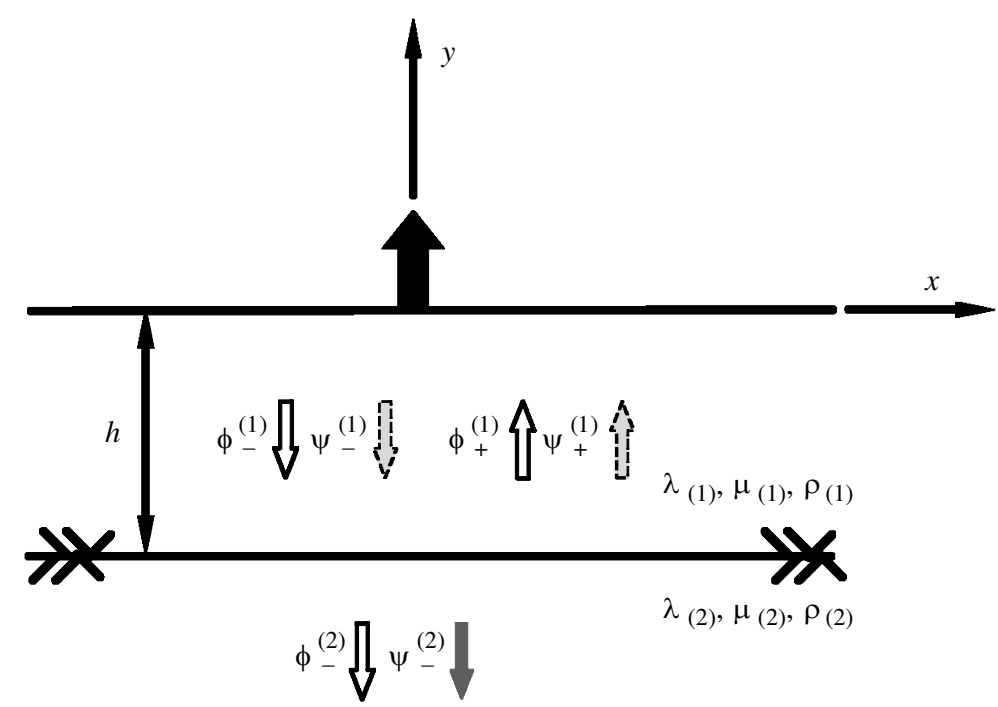

Figure 2. The coordinate system and the geometry of one layer of elastic solid overlying a half-space.

integration through the mapping in (2.7), as discussed by Pao \& Gajewski (1977). The contour integration is evaluated by 24-point Gaussian integration formulation with constant time-step $\Delta t$, and the convolution integral in (2.9) is computed directly by using a discrete convolution algorithm.

The transient solution can be obtained by inverting the transformed solution in (2.3) with the aid of (2.9). Each term in (2.3) represents a physical transient wave. Since each wave has its own arrival time, only a finite number of terms are involved in the early-time solution. There is a leading wave in each group of waves which propagates fastest in the layered medium; the total number of groups which arrives at the receiver during the observation time can be obtained in advance. Therefore, a truncation criterion of the infinite series solution in (2.3) is suggested. A synthesis of all waves arriving at the receiver during the observation time leads to the transient responses of the layered medium. In the following sections, the numerical calculations for the transient response of a two-layered medium (a layer overlying a half-space) subjected to a surface dynamic loading will be discussed in detail.

\section{Transient responses for a two-layered medium}

In this section, the transient responses of a layer with thickness $h$ overlying a halfspace and subjected to dynamic concentrated loadings are analysed in detail. Let the densities and Lamé constants in the layer and half-space be denoted by $\rho_{1}, \lambda_{1}$ and $\mu_{1}$ and $\rho_{2}, \lambda_{2}$ and $\mu_{2}$, respectively. At time $t=0$, an in-plane concentrated vertical force with constant magnitude $\sigma_{0}$ is applied suddenly on the top surface of the layer (1), as shown in figure 2. The time dependence of loadings is represented by the Heaviside step-time function $H(t)$, which is chosen as the Green's function. For any arbitrary time dependence, the response can be obtained by the convolution theorem or Duhamel's integral. 
In the two-layered medium, the global field vector is a six-element vector and is expressed as follows:

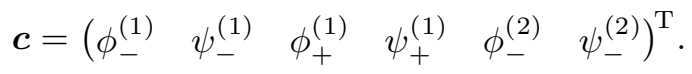

The global applied displacement-traction vector is determined by applied loadings on the top surface, and is given by

$$
\boldsymbol{t}=\left(\begin{array}{llllll}
\sigma_{0} H(t) & 0 & 0 & 0 & 0 & 0
\end{array}\right)^{\mathrm{T}} .
$$

Thus the source vector is obtained by eqn (4.17) of part I as follows,

$$
\boldsymbol{s}=\left(\frac{\sigma_{0}}{p^{3} \mu_{1}} \frac{s_{\mathrm{T}(1)}^{2}-2 \xi^{2}}{R(\xi)} \quad \frac{-\sigma_{0}}{p^{3} \mu_{1}} \frac{2 \xi \gamma_{\mathrm{L}(1)}}{R(\xi)} \quad 0 \quad 0 \quad 0 \quad 0 \quad 0\right)^{\mathrm{T}}
$$

where

$$
R(\xi)=\left(s_{\mathrm{T}(1)}^{2}-2 \xi^{2}\right)^{2}+4 \xi^{2} \gamma_{\mathrm{L}(1)} \gamma_{\mathrm{T}(1)} .
$$

Equation (3.4) is the characteristic equation for Rayleigh surface waves which propagate non-dispersively along the free surface.

The phase-related reflection and transmission matrix $\boldsymbol{R}$ is independent of the loading conditions, and is represented by a $6 \times 6$ matrix as follows,

$$
\begin{aligned}
& \boldsymbol{R}=\left[\begin{array}{cc}
0 & 0 \\
0 & 0 \\
\left(R_{p p}\right)_{1 / 2} \mathrm{e}^{-2 p \gamma_{\mathrm{L}}^{(1)} h} & \left(R_{s p}\right)_{1 / 2} \mathrm{e}^{-p\left(\gamma_{\mathrm{L}}^{(1)}+\gamma_{\mathrm{T}}^{(1)}\right) h} \\
\left(R_{p s}\right)_{1 / 2} \mathrm{e}^{-p\left(\gamma_{\mathrm{L}}^{(1)}+\gamma_{\mathrm{T}}^{(1)}\right) h} & \left(R_{s s}\right)_{1 / 2} \mathrm{e}^{-2 p \gamma_{\mathrm{T}}^{(1)} h} \\
0 & 0 \\
0 & 0
\end{array}\right. \\
& \left.\begin{array}{cccc}
\left(R^{p p}\right)_{1 / 0} & \left(R^{s p}\right)_{1 / 0} & 0 & 0 \\
\left(R^{p s}\right)_{1 / 0} & \left(R^{s s}\right)_{1 / 0} & 0 & 0 \\
0 & 0 & 0 & 0 \\
0 & 0 & 0 & 0 \\
\left(T_{p p}\right)_{1 / 2} \mathrm{e}^{-p\left(\gamma_{\mathrm{L}}^{(1)}-\gamma_{\mathrm{L}}^{(2)}\right) h} & \left(T_{s p}\right)_{1 / 2} \mathrm{e}^{-p\left(\gamma_{\mathrm{T}}^{(1)}-\gamma_{\mathrm{L}}^{(2)}\right) h} & 0 & 0 \\
\left(T_{p s}\right)_{1 / 2} \mathrm{e}^{-p\left(\gamma_{\mathrm{L}}^{(1)}-\gamma_{\mathrm{T}}^{(2)}\right) h} & \left(T_{s s}\right)_{1 / 2} \mathrm{e}^{-p\left(\gamma_{\mathrm{T}}^{(1)}-\gamma_{\mathrm{T}}^{(2)}\right) h} & 0 & 0
\end{array}\right],
\end{aligned}
$$

where the reflection coefficients at the free surface $y=0, R^{p p}, \ldots, R^{s s}$, are given in eqn (5.13) of part I by setting $\mu_{0}=0$, and the reflection and transmission coefficients at $y=-h$ are given in eqn (5.5) of part I. Since no wave comes from infinity to the interface, there will be zero eigenvalue for the matrix $\boldsymbol{R}$. Furthermore, when the transform parameter $p$ is sufficiently small, all eigenvalues of $\boldsymbol{R}$ will be located within a unit circle on the complex $\xi$-plane. The convergence of the matrix Bromwich expansion is thus ensured.

If we are concerned only with displacements $u$ and $v$ and the stress components $\sigma_{y y}$ and $\sigma_{x y}$ in layer (1), then the response vector is chosen as

$$
\hat{\boldsymbol{b}}(y)=\left(\begin{array}{llll}
\hat{u}^{(1)} & \hat{v}^{(1)} & \hat{\sigma}_{y y}^{(1)} & \hat{\sigma}_{x y}^{(1)}
\end{array}\right)^{\mathrm{T}},
$$




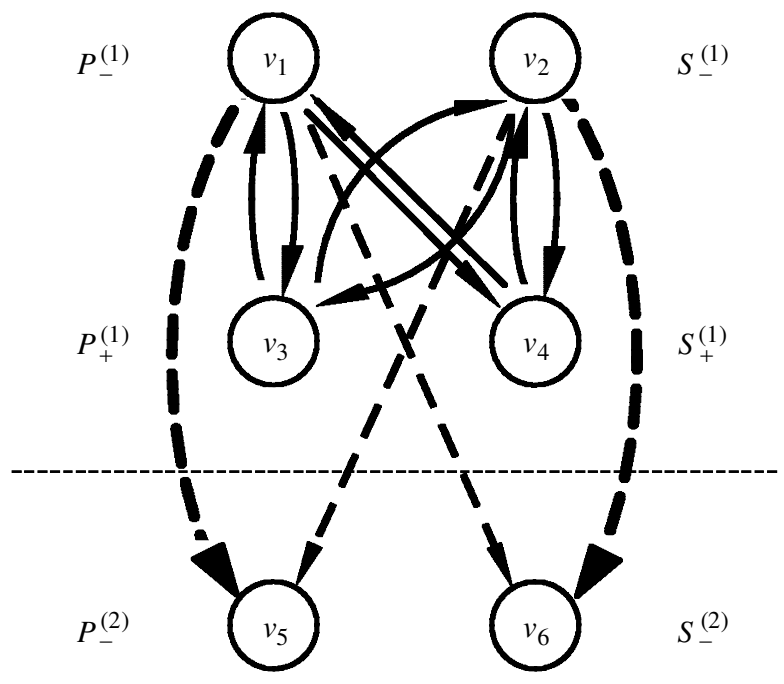

Figure 3. The graphic representation of the reflection and transmission matrix $\boldsymbol{R}$.

and the receiver matrix $\boldsymbol{R}_{\boldsymbol{c} \boldsymbol{v}}$ is a $4 \times 6$ matrix expressed as follows

$$
\boldsymbol{R}_{\boldsymbol{c} \boldsymbol{v}}(y)=\left[\begin{array}{lll}
\boldsymbol{M}_{11}^{(1)}(y) & \boldsymbol{M}_{12}^{(1)}(y) & \mathbf{0} \\
\boldsymbol{M}_{21}^{(1)}(y) & \boldsymbol{M}_{22}^{(1)}(y) & \mathbf{0}
\end{array}\right],
$$

where $\mathbf{0}$ is a $2 \times 2$ zero matrix and $\boldsymbol{M}_{11}^{(1)}, \ldots, \boldsymbol{M}_{22}^{(1)}$ are given in eqn (3.8) of part I. The receiver matrix $\boldsymbol{R}_{\boldsymbol{c} \boldsymbol{v}}$ depends only on the response vector, and again is independent of the loading conditions.

The matrix solution in the transformed domain is determined and is given in eqn (4.21) of part I as follows:

$$
\hat{\boldsymbol{b}}(y ; \xi, p)=\boldsymbol{R}_{\boldsymbol{c} \boldsymbol{v}}(y) \sum_{i=0}^{\infty} \boldsymbol{R}^{i} \boldsymbol{s} .
$$

The transient solutions in time domain for response vector $\boldsymbol{b}$ are obtained by sorting the matrix solution into a series form and applying Cagniard's method, as the procedure stated in the previous section. Once the solution for the step-time response is obtained, another type of source-time function can be obtained by a convolution integral.

To show the relations between the matrix solution and the conventional generalized ray solution, the physical interpretations of the matrices $\boldsymbol{R}$ and $\boldsymbol{R}^{i}$ are considered in detail through the example for the two-layered medium. The phase-related reflection and transmission matrix $\boldsymbol{R}$ can be regarded as the 'adjacency matrix' of a graph, which stores the transfer functions relating each propagating mode. To explain this, six points $v_{1}, v_{2}, v_{3}, v_{4}, v_{5}$ and $v_{6}$ were used to represent the propagating modes

$$
p_{-}^{(1)}, \quad s_{-}^{(1)}, \quad p_{+}^{(1)}, \quad s_{+}^{(1)}, \quad p_{-}^{(2)}, \quad s_{-}^{(2)},
$$

respectively, as shown in figure 3 . In the graphic representation, $p_{+}^{(1)}$ and $s_{+}^{(1)}$ represent the wave potentials for the $\mathrm{P}$ - and $\mathrm{S}$-wave propagating in the direction of 
Table 1. Physical interpretation of entries of matrix $\boldsymbol{R}^{i}$

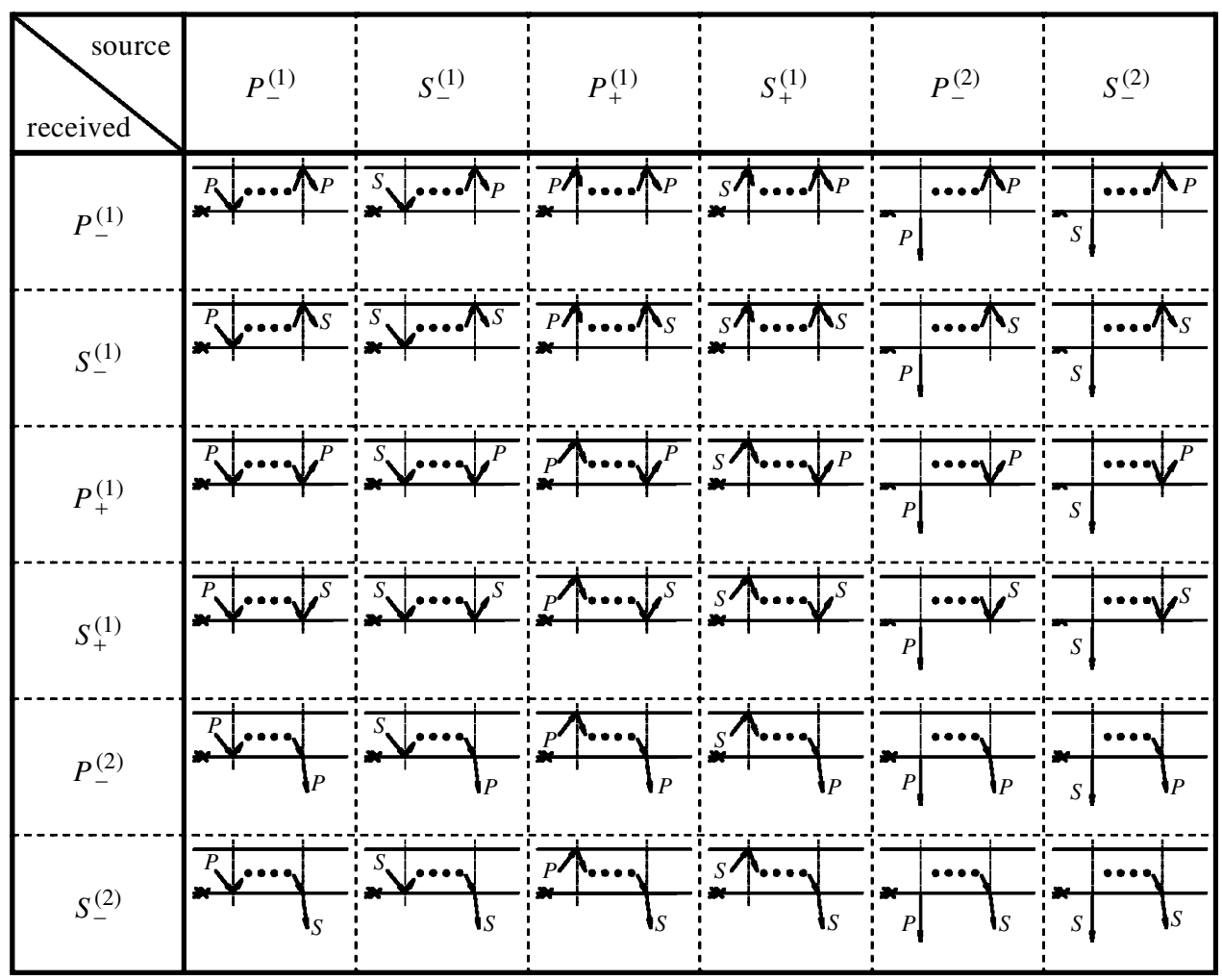

increasing $y$ within layer (1). When reflection or transmission occurs, the waves will change their propagating modes. The adjacency matrix $\boldsymbol{R}$ records the transfer relations between the propagating modes. If the $(i-j)$ entry of $\boldsymbol{R}$ is zero, it means the transfer of propagating mode from $v_{i}$ to $v_{j}$ after one reflection is impossible. Furthermore, if the wave starting with propagating mode $v_{i}$ generates a wave with mode $v_{j}$ after it is reflected by, or transmitted through, the interfaces $i$ times, all offspring of this wave can be found in the $(i-j)$ entry of $\boldsymbol{R}^{i}$. The physical interpretations for each entry of the power matrix $\boldsymbol{R}^{i}$ are made and summarized in table 1 . A zero entry in matrix $\boldsymbol{R}^{i}$ implies there is a physically impossible ray path after $i$ reflections. The relationship between generalized ray theory and the matrix solution is thus established.

\section{Numerical results of transient responses for a two-layered medium}

In the following numerical examples, the transient responses of a solid layer with thickness $h$ overlying a solid half-space and subjected to a vertical concentrated force at the upper surface of the layer are discussed. The step-time response was calculated first and the geometric configuration is shown in figure 2. While the arbitrary sourcetime function is involved, the discrete convolution algorithm is used to obtain the corresponding transient response. In evaluating integrals on the complex $\xi$-plane given by (2.9), the Gaussian quadratures with 24 points were used. 


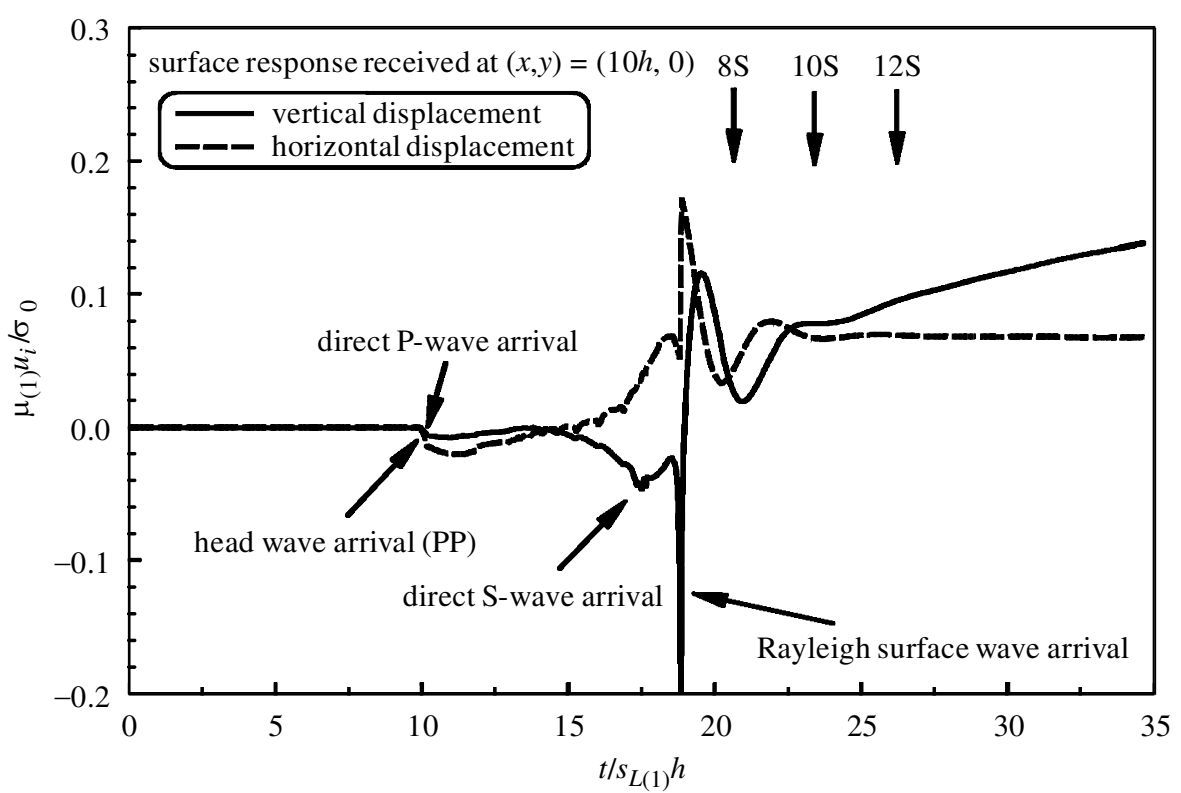

Figure 4. Vertical and horizontal displacement responses at $(x, y)=(10 h, 0)$ on the free surface of the two-layered medium subjected to a point force.

The transient response of vertical and horizontal displacements at a distance $10 \mathrm{~h}$ from the applied loading on the free surface of the two-layered medium is shown in figure 4; the material constants were assumed to be (Pekeris et al. 1965)

$$
\begin{aligned}
& \lambda_{1}=\mu_{1}, \quad \rho_{1}=1.21, \quad c_{s(1)}=1.0, \quad c_{p(1)}=\sqrt{3} c_{s(1)}, \\
& \mu_{2}=2 \mu_{1}, \quad \rho_{1}=2.0, \quad c_{s(2)}=1.1, \quad c_{p(2)}=\sqrt{3} c_{s(2)} .
\end{aligned}
$$

The numerical calculation based on above material constants is equal to the use of non-dimensional time $\tau=t / s_{\mathrm{L}(1)} h$, providing that $h=1$. Note that the nondimensional observation time was up to $\tau=35$ in the figure, of which 35 groups of waves were induced and 1146 distinct waves are calculated. However, the total number of waves generated during the observation time was about $4.5 \times 10^{7}$. The degeneration problem becomes more serious when the observation time is longer. Though the number of terms in eqn (4.21) of part I is infinity, only a finite number of terms will be non-zero in the series' solution during a finite observation time. In the calculated case (as shown in figure 4), the groups of waves after 35 reflections or transmissions did not occur and their contributions to the waveform are zero during the observation time.

Since the lower medium has higher wave speeds, waves in the overlying medium may propagate partly along the interface with the wave speed of the lower medium and may arrive earlier than those propagating within the slower speed medium. The first signal arriving at $\tau=9.94$ in figure 4 was generated by a PP-wave which refracted along the interface. However, the direct $\mathrm{P}$-wave arrived at $\tau=10$. The criterion of the possibility of head waves can be determined mathematically or physically, and is discussed in detail in the review article by Pao \& Gajewski (1977). 


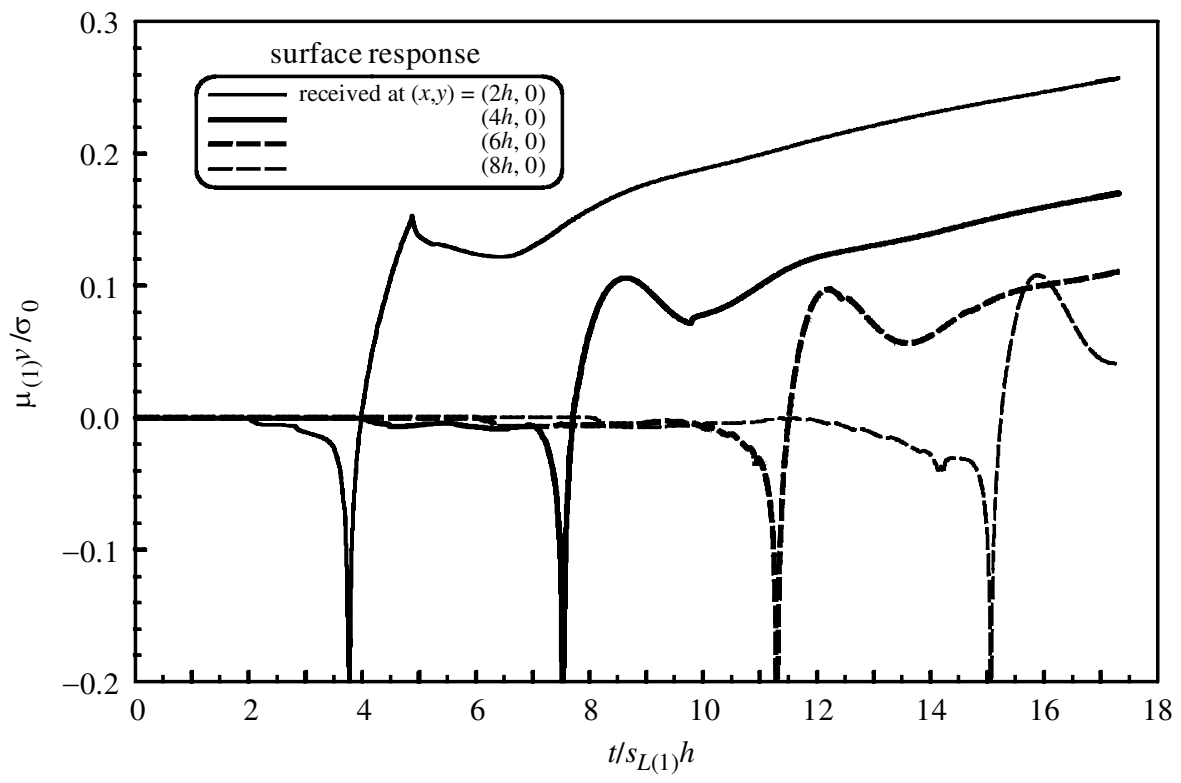

Figure 5. The transient responses of vertical displacement on the free surface at distances $2 h$, $4 h, 6 h$ and $8 h$ from the applied loading.

The first arrival signal of the horizontal displacement is greater than that of the vertical displacement, as represented in figure 4. The strongest motion of the free surface shown in figure 4 can be easily identified as the Rayleigh surface wave arrival at the material point. Since the surface concentrated force with a step-time function is applied, the vertical displacement will approach infinity when the Rayleigh wave arrives, and the horizontal displacement responds to a discontinuity at the same time. The non-dispersion property of the Rayleigh surface wave is shown in figure 5 , in which the receivers are located at the free surface with distances $x=2 h, 4 h, 6 h$ and $8 h$ from the applied loading. The Rayleigh surface wave propagated with a constant speed $c_{\mathrm{R}} \approx 0.92 c_{\mathrm{s}}$. The slowness of the Rayleigh surface wave can be determined directly by solving the characteristic equation of Rayleigh waves, $R(\xi)=0$, given in (3.4). On the other hand, the discontinuity in the slope shown in the figure is caused by the arrival of waves which carry singularities at the wave front, as discussed in $\S 2$.

The singularities in displacements and stresses are mainly caused by the Heaviside source-time function of the loading. It can be reduced by changing the source-time function into the rounded-shoulders Heaviside function, as shown in figure 6 . The width $2 \Delta$ is a measure of the sharpness of the pulse. In the limit of vanishing $\Delta$, the function approaches the Heaviside unit function, and its derivative behaves as a $\delta$ function. The time histories of vertical and horizontal velocities of the material point on the free surface $10 \mathrm{~h}$ away from the loading are obtained by the convolution theorem and the results are shown in figure 7. The rounded-shoulder Heaviside source function with arising time $\Delta=0.34$ was used in this calculation. While the rounded-shoulder Heaviside source-time function is involved, the arrival of the strongest response caused by the Rayleigh wave will be delayed by $\Delta$.

The transient responses of stress components at the mid-plane of the overlying layer with horizontal distance $x=5 h$ are shown in figure 8 . It shows that the stress 


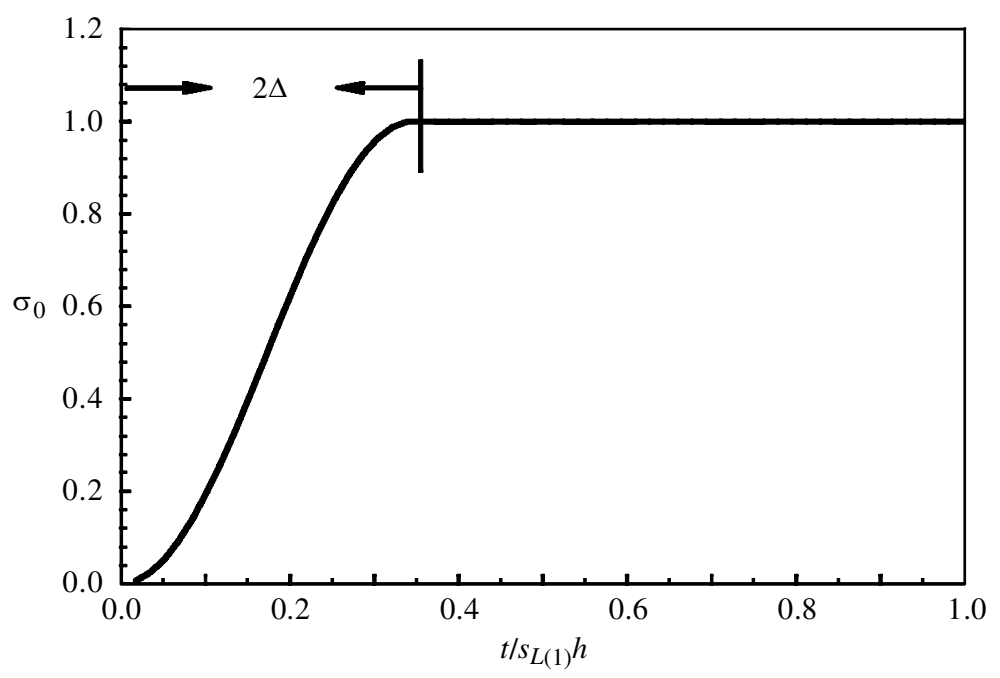

Figure 6. The rounded-shoulder Heaviside function.

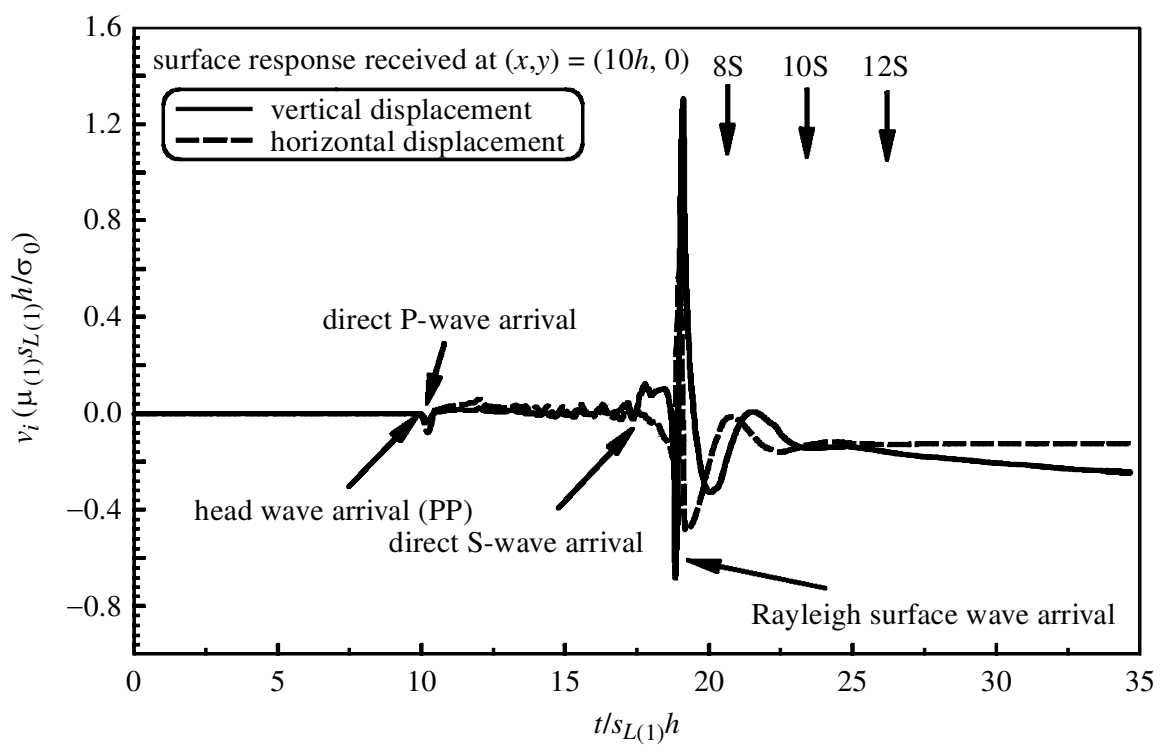

Figure 7. Vertical and horizontal velocities at $(x, y)=(10 h, 0)$ on the free surface of the two-layered medium.

approaches the correspondent static value after the dominant waves passed by. The maximum amplitude occurs at $\tau=9.5$, and the responses approach the static values after $\tau=14$. It should be noted that the largest stress component is $\sigma_{x x}$, while the stresses $\sigma_{y y}$ and $\sigma_{x y}$ will decay as the time value increases. It is of interest to note that each term in (2.3) diverges as time approaches infinity, but the sum will converge to a static value if the applied force is a Heaviside or rounded-shoulder Heaviside time dependence. Furthermore, in this analysis, the transient response was decomposed into many convergent groups in which waves of the same number of reflections were summed. 


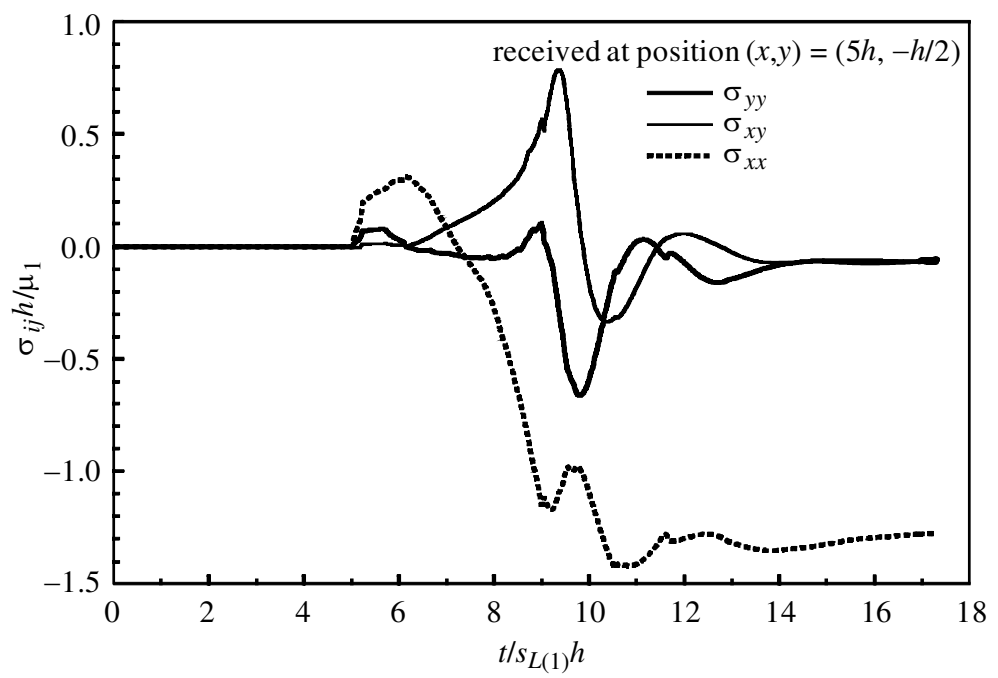

Figure 8 . The time history of transient response for stresses at $(x, y)=\left(5 h,-\frac{1}{2} h\right)$.

The solid-solid interface problem has been discussed in great detail by Cagniard (1939). The domain of existence of a Stoneley wave (an interface wave) was investigated extensively. The characteristic equation for the Stoneley wave is given in eqn (5.9) of part I. It is well known that an interface pulse (a Stoneley wave) can exist for the joint half-space, but only for a very restricted class of solid pairs. The conditions for the existence of Stoneley waves when the Poisson ratio of both solids is equal to 0.25 were discussed by Cagniard (1939). Figure 9 shows the vertical displacements of material points at $x=2 h, 4 h, 6 h$ and $8 h$ along the interface. Although the combination of material properties for both solids used in this numerical simulation is located beyond the domain of existence of Stoneley wave, there exists an almost non-dispersive wave propagating along the interface. However, Gilbert \& Laster (1962) suggested that there are Stoneley-like pulses (sometimes called pseudoStoneley waves) propagating along the interface of two dissimilar media with parameter values outside the classic Stoneley wave region of existence for almost all solid pairs. Figure 9 shows the characteristics of Stoneley-like pulses. On the other hand, the vertical displacements along the interface for the media with parameter values inside the region of existence of Stoneley waves were also investigated in this study, and the results are indicated in figure 10. The non-dispersion property of Stoneley waves is shown clearly in this figure.

\section{Experimental measurements on transient waves in a two-layered medium}

An experimental system was set up to study the transient waves propagating on a two-layered medium. The measured vertical displacements on free surface of a twolayered specimen were compared with the numerical calculations. In the experiments of transient elastic waves propagation, NBS conical transducers were used to measure the transient vertical surface displacement signals in a specimen. Since the tip diameter of the NBS transducer is $1.5 \mathrm{~mm}$, it can be approximated as a point receiver. The received voltage signals from the NBS transducer were fitted in a pre-amplifier 


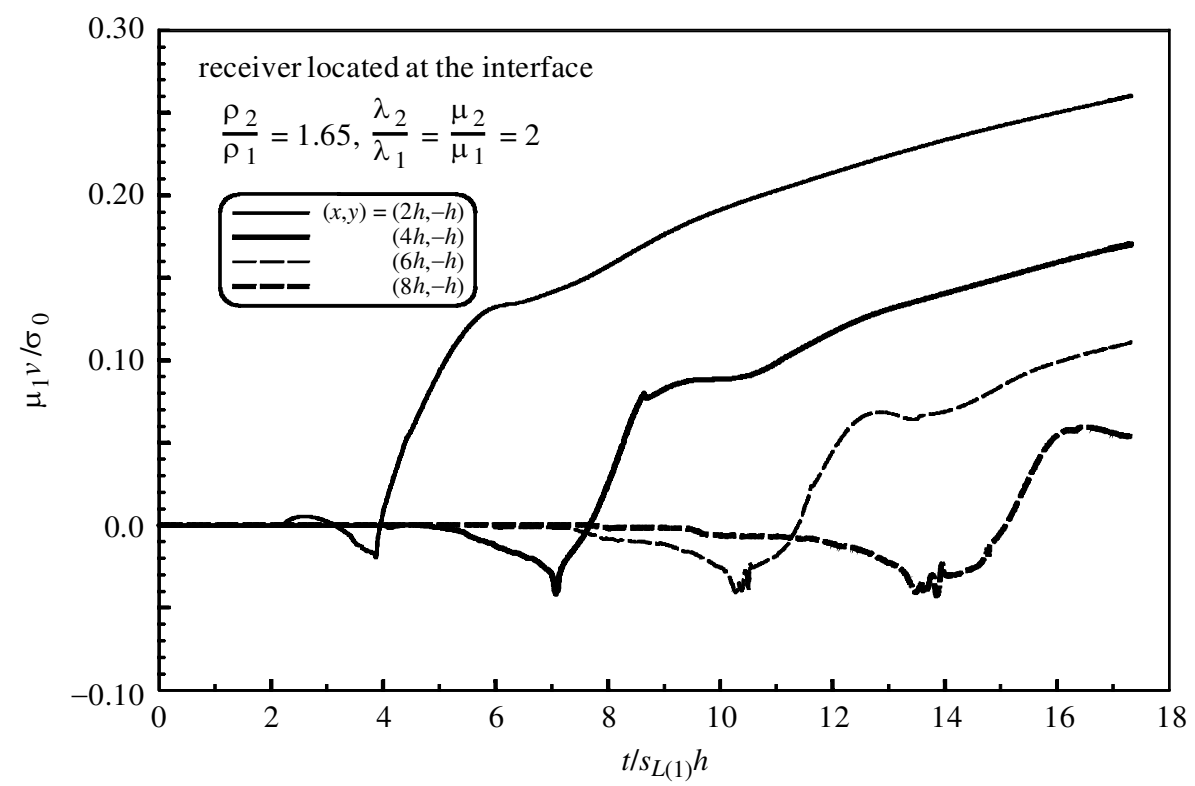

Figure 9. The vertical displacement received at various material points with horizontal distances $2 h, 4 h, 6 h$ and $8 h$ from the applied loading along the interface (the physical parameters lie outside the region determined by the Stoneley equation).

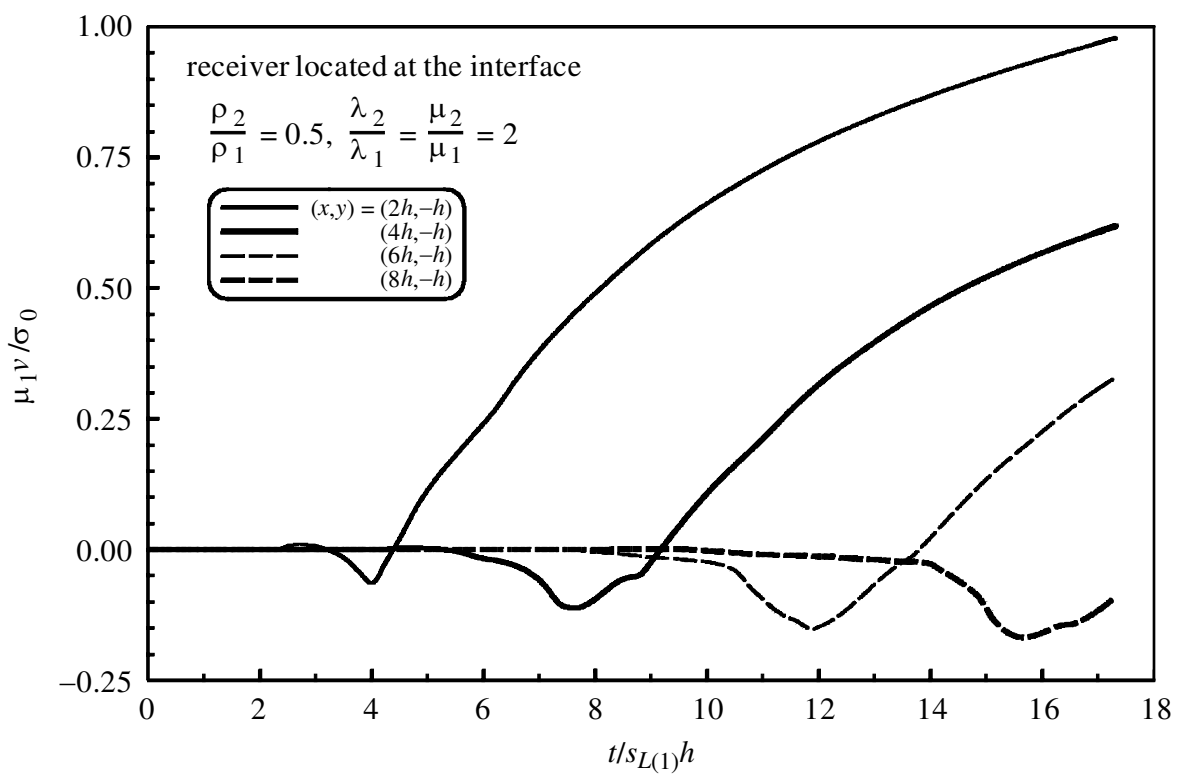

Figure 10. The vertical displacement received at various material points with horizontal distances $2 h, 4 h, 6 h$ and $8 h$ from the applied loading along the interface (the physical parameters lie inside the region determined by the Stoneley equation). 


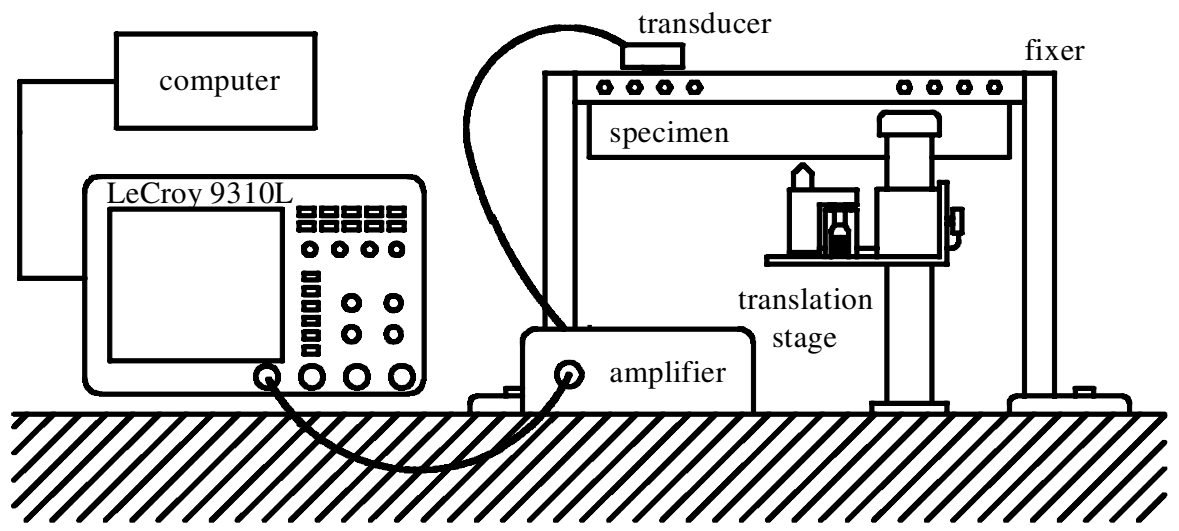

Figure 11. The system of two-dimensional acoustical emission experiment.

Table 2. Geometric configurations of specimens ( $\mathrm{mm})$

number specimen (upper-lower layer)

$\begin{array}{ll}1 & \text { aluminium-aluminium } \\ 2 & \text { aluminium-stainless steel } \\ 3 & \text { brass-aluminium } \\ 4 & \text { acrylic-aluminium } \\ 5 & \text { aluminium }\end{array}$

5 aluminium dimension of upper/lower layer

$$
\begin{gathered}
300 \times 16.05 \times 1.2, \quad-300 \times 150 \times 1.2 \\
300 \times 14.80 \times 1.2, \quad-300 \times 150 \times 1.2 \\
300 \times 14.75 \times 1.2, \quad-300 \times 150 \times 1.2 \\
300 \times 13.75 \times 1.2, \quad-300 \times 150 \times 1.2 \\
300 \times 150 \times 1.2
\end{gathered}
$$

and recorded by a digital oscilloscope (Lecory 9314L, the highest sampling rate is $100 \mathrm{Ms} \mathrm{s}^{-1}$ and the vertical resolution is $\left.8 \mathrm{bits}\right)$. The experimental measurement system is shown in figure 11 .

Transient elastic wave experiments were conducted on thin plates of thicknesses $1 \mathrm{~mm}$, which are composed of an overlying strip and a large plate. The interfaces were bonded with epoxy resin (PC-10 adhesive) for a curing time of $c a .1 \mathrm{~h}$ under pressure produced by a vice. The bonding surfaces of thin plates were polished carefully to ensure perfect contact at the interface. Five specimens were used to measure the vertical displacements at different point on the free surface. They were made of aluminium-aluminium, aluminium-stainless steel, brass-aluminium and acrylicaluminium, numbered 1, 2, 3 and 4, respectively. In addition, a specimen numbered 5 was prepared to perform a control experiment for checking the bonding condition on the interface. The dimensions of the bed plates are large enough to simulate the half-space during the observation time. The dimensions of the five specimens are listed in table 2. The densities and the elastic constants of the brass, stainless steel, aluminium and acrylic thin plates are given in table 3 .

Since the experiments were conducted on thin plates, the plane stress wave propagation is assumed in our numerical calculation. The solutions for plane stress conditions are the same as those for the plane strain case discussed in previous sections, provided that the constant $\lambda$ is replaced by $\lambda^{*}=2 \mu \lambda /(\lambda+2 \mu)$. The $\mathrm{P}$-wave speed for the plane stress case is decreased, while the $\mathrm{S}$-wave speed is the same as that for the plane strain case. However, the speed of the Rayleigh wave is changed slightly because it depends strongly on the speed of the S-wave. 
Table 3. Material constants of the specimens

\begin{tabular}{lccccc}
\hline material & $\rho\left(\mathrm{kg} \mathrm{m}^{-3}\right)$ & $E(\mathrm{GPa})$ & $\nu$ & $c_{\mathrm{L}}\left(\mathrm{m} \mathrm{s}^{-1}\right)$ & $c_{\mathrm{T}}\left(\mathrm{m} \mathrm{s}^{-1}\right)$ \\
\hline brass & 8600 & 1100 & 0.34 & 4437 & 2184 \\
stainless steel & 7850 & 207 & 0.29 & 5878 & 3197 \\
aluminium & 2700 & 70 & 0.33 & 6197 & 3121 \\
acrylic & 1190 & 6.32 & 0.32 & 2717 & 1426 \\
\hline
\end{tabular}

The concentrated force applied on the top surface of the medium with an approximate step source-time function can be generated by the breakage of pencil leads (Sachse \& Hsu 1979). The source-time function shown in figure 12 was obtained by breaking the pencil lead directly on the NBS conical transducer. The source-time function generated by the breakage of pencil leads has a short rising time of $c a .1 \mu \mathrm{s}$ and is reproducible without damaging the sample. Note that the source-time function received by the transducer decays exponentially as time increases. The exponential decay is caused by the dynamic characteristic of the NBS conical transducer. However, a function can be used to fit the source-time function as

$$
f(t)=\mathrm{e}^{-\alpha t}\left(1-\left(1-\beta t^{2}\right) \mathrm{e}^{-\varsigma t^{2}} \cos \chi t\right) H(t),
$$

where the parameter $\alpha$ dominates the effect of exponential decay caused by the conical transducer, $\chi$ and $\zeta$ characterize the ripples and overshoot on the sourcetime curve, and $\beta$ controls the minor characteristic of the curve. The parameters given in figure 12 can fit the source-time function well, at least up to $250 \mu$ s. Note that the source-time function in (5.1) is twice differentiable, and the first derivative evaluated at the origin is zero, which satisfies the condition assumed in (2.9). The numerical calculation was thus convoluting with (5.1) to reduce the error induced by the source-time functions together with the sensor system.

To check the bonding effects of using epoxy resin, a specimen which was composed of two aluminium plates with dimensions listed in table 2 (specimen number 1) was prepared. If the specimen is bonded perfectly, the response will behave like a halfspace. The experimental results for specimens 1 and 5, as well as the numerical calculations, are illustrated in figure 13. The vertical displacements were measured and calculated at the free surface with a distance of $6 \mathrm{~cm}$ away from the source. Each experimental waveform was obtained by averaging ten measurements to reduce the noises. Since the magnitude of the source function was unknown, the experimental results were scaled by a suitable factor to fit the numerical calculation. Due to the finite geometry of the specimen, the waves reflected from the side edge arrive at the receiver during the observation time. Thus, the theoretical waveform is valid only before the arrival of side waves. The comparisons between theoretical and experimental waveforms (shown in figure 13) are in good agreement before the arrival of side waves. Furthermore, the good agreement between the experimental waveforms for specimens 1 and 5 implies that the bonding condition by epoxy resin is nearly perfect.

The experimental waveforms and the numerical calculations for the vertical displacements on the free surface of specimen 2 with a distance of $3,6,9$ and $12 \mathrm{~cm}$ away from the location of the applied force are shown in figure 14. The numerical calculations based on the analytical solutions agree very well with the experimental 


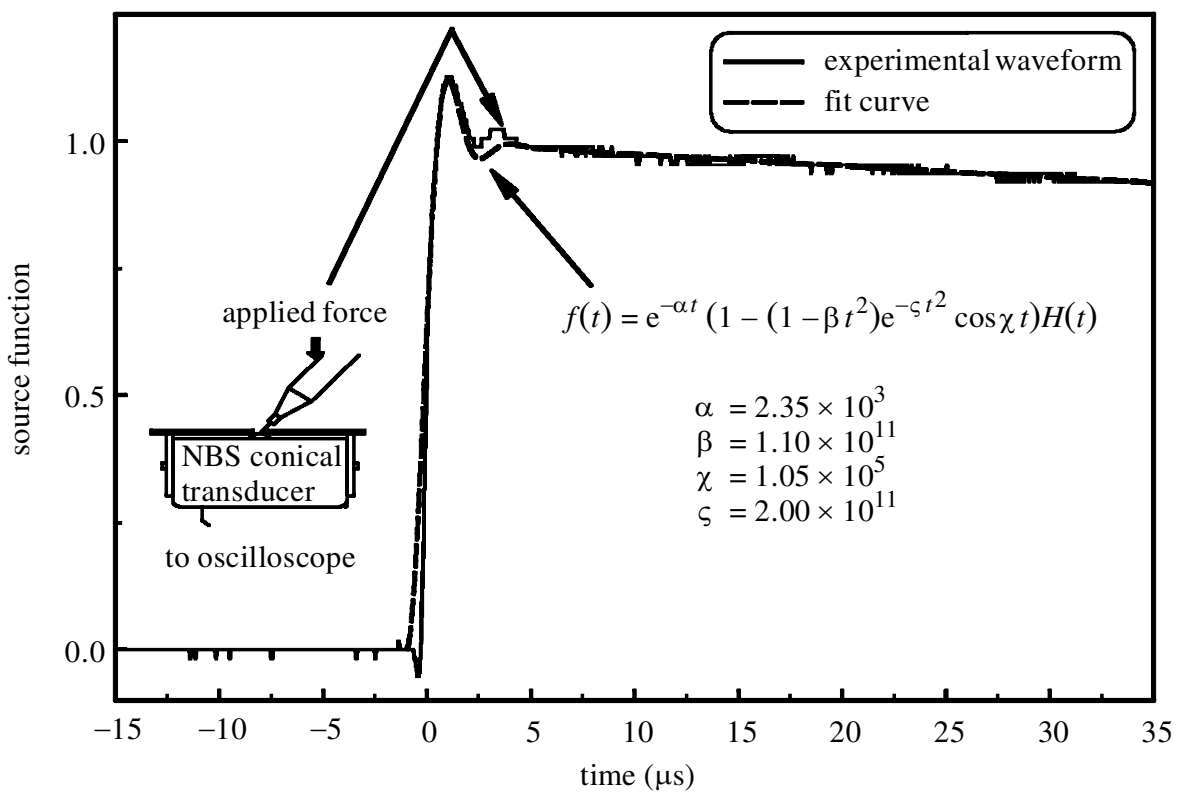

Figure 12. The calibration experiment for pencil lead breaking source and NBS conical transducer.

waveforms in the early-time responses. However, the numerical calculations fail to fit the experimental waveform at $c a .55 \mu \mathrm{s}$, since the reflected waves from the side edges, which are not taken into account in the analytical model, have arrived at the receiver. For specimen 2, it is noted that the wave speed relation between the overlying layer and the half-space is $c_{p(1)}>c_{p(2)}>c_{s(2)}>c_{(1)}$. The non-dispersive property of Rayleigh surface waves can be identified in the figure. The oscillations, with slight damping of vertical displacement, are found in this figure. Soon after the arrival of the Rayleigh surface wave, the surface responses of this specimen behave like those for the half-space. If the distance between source and receiver is larger, the damping of the oscillation after the arrival of the Rayleigh wave will be smaller.

Figure 15 shows the experimental waveforms for the vertical displacements on the free surface of specimen 3. It is noted that the wave speed relation between the overlying layer and the half-space is $c_{p(2)}>c_{p(1)}>c_{s(2)}>c_{(1)}$. The transient response of this specimen behaves like the surface vertical displacement for a halfspace. However, the surface response is smaller than that of the half-space because of the support of the bed half-space. Generally speaking, the acoustic impedance is an index for reflection and transmission coefficients for normal incidents of pressure waves towards an interface. When the acoustic impedances of the upper layer and the bed half-space are very close, the surface responses near the vertical point force will behave like a half-space, since the reflection coefficient almost vanishes. Specimen 3 consists of aluminium and brass, with acoustic impedances of $1.7 \times 10^{7}$ and $2.1 \times$ $10^{7} \mathrm{~kg} \mathrm{~m}^{-2} \mathrm{~s}^{-1}$, respectively. In comparison to specimen 2 , which is composed of aluminium and stainless steel, with acoustic impedances of $1.7 \times 10^{7}$ and $4.5 \times$ $10^{7} \mathrm{~kg} \mathrm{~m}^{-2} \mathrm{~s}^{-1}$, the near-field response of specimen 3 should behave like a half-space.

Figure 16 is the transient vertical displacement signal received at $x=3 \mathrm{~cm}$ away from the applied loading on the free surface of specimen 4 . The numerically calculated 

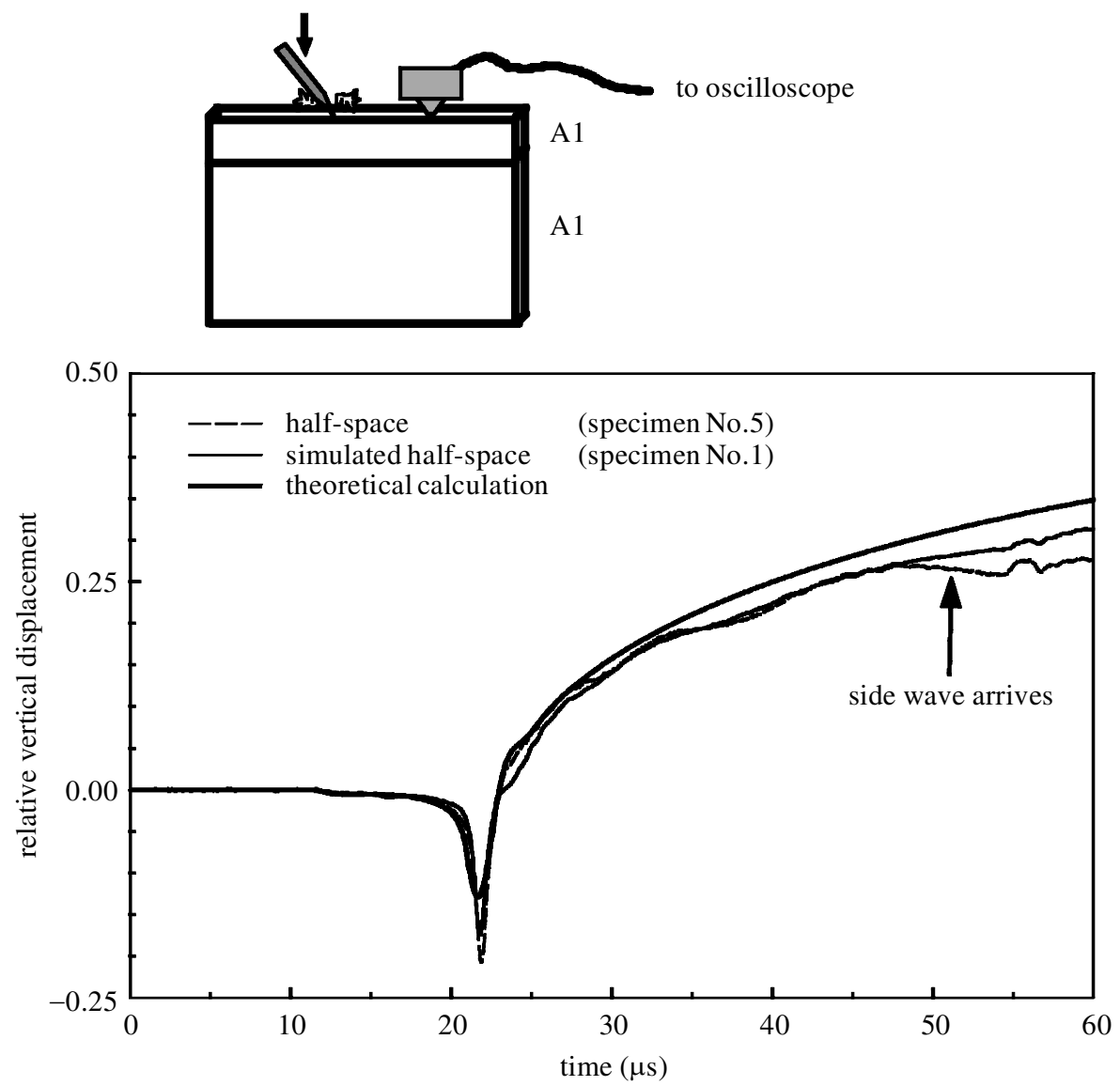

Figure 13. The control experiment for the bonding condition.

and experimentally measured results are in agreement at the early-time response. The numerical results cannot fit the experimental measurement well after the arrival of a Rayleigh wave. It is possibly caused by the anelastic nature of acrylic and the effect of the bonding layer. The numerically calculated time history of vertical displacement shows a periodical oscillation with damping at almost a constant value.

The ratio of Young's modulus for the upper and the lower materials of specimen 4 is about 11; the bed half-space can be regarded as a rigid body-like material. If the interface is a perfect rigid surface, the time history of the vertical displacement for the free surface of the layer will oscillate periodically, with slight attenuation around the static value (see figure 17). When the bed half-space is replaced by an elastic solid, the transient responses show quite different characteristics, as indicated in figures $14-16$.

From the results shown in figures 14-16, it is found that the propagation of acoustic emission waves in a perfectly bonded layered half-space can be simulated very well by both numerical and experimental approaches. From the measured signal, the relative material properties of the upper layer and the lower bed half-space can be estimated. The agreement between numerically calculated and experimentally measured wave responses implies the possible application of the transient elastic 


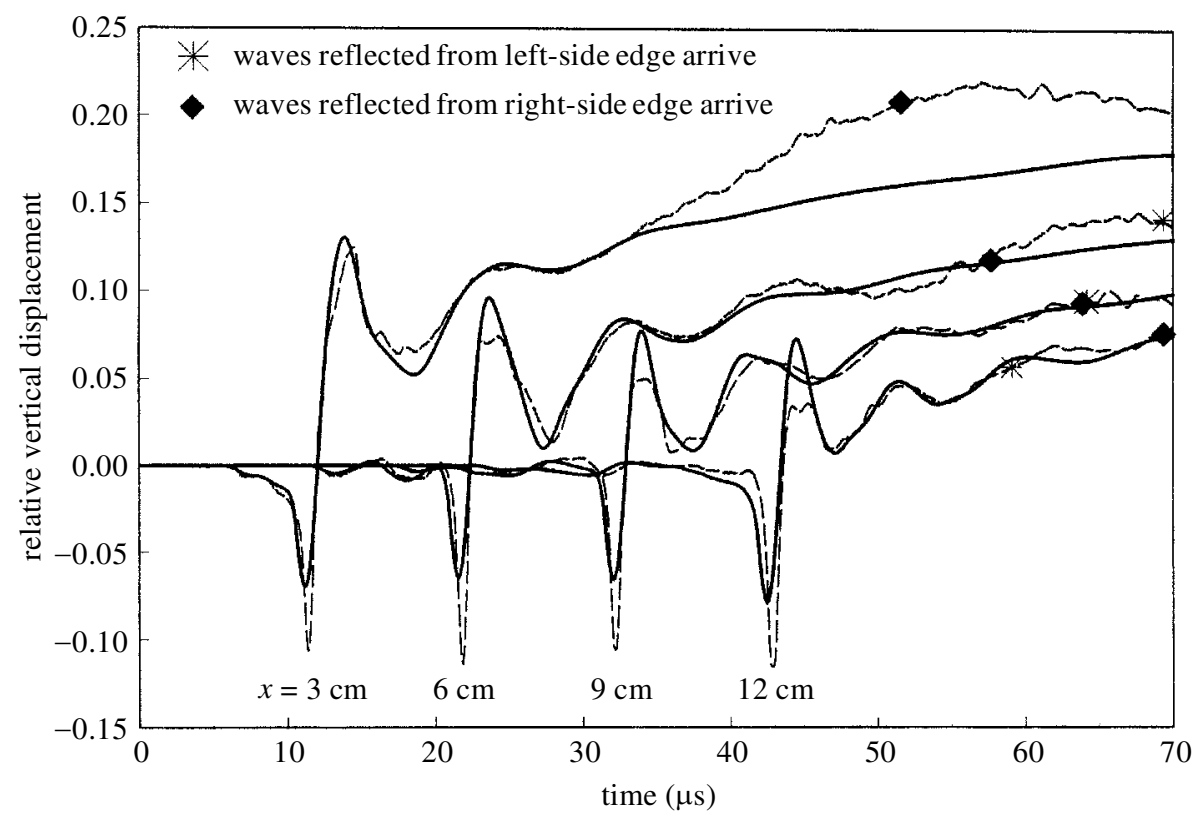

Figure 14. Comparison of measured and calculated waveforms on the free surface of specimen 2 (dashed lines for the experimental measurements, solid lines for the numerical calculations).

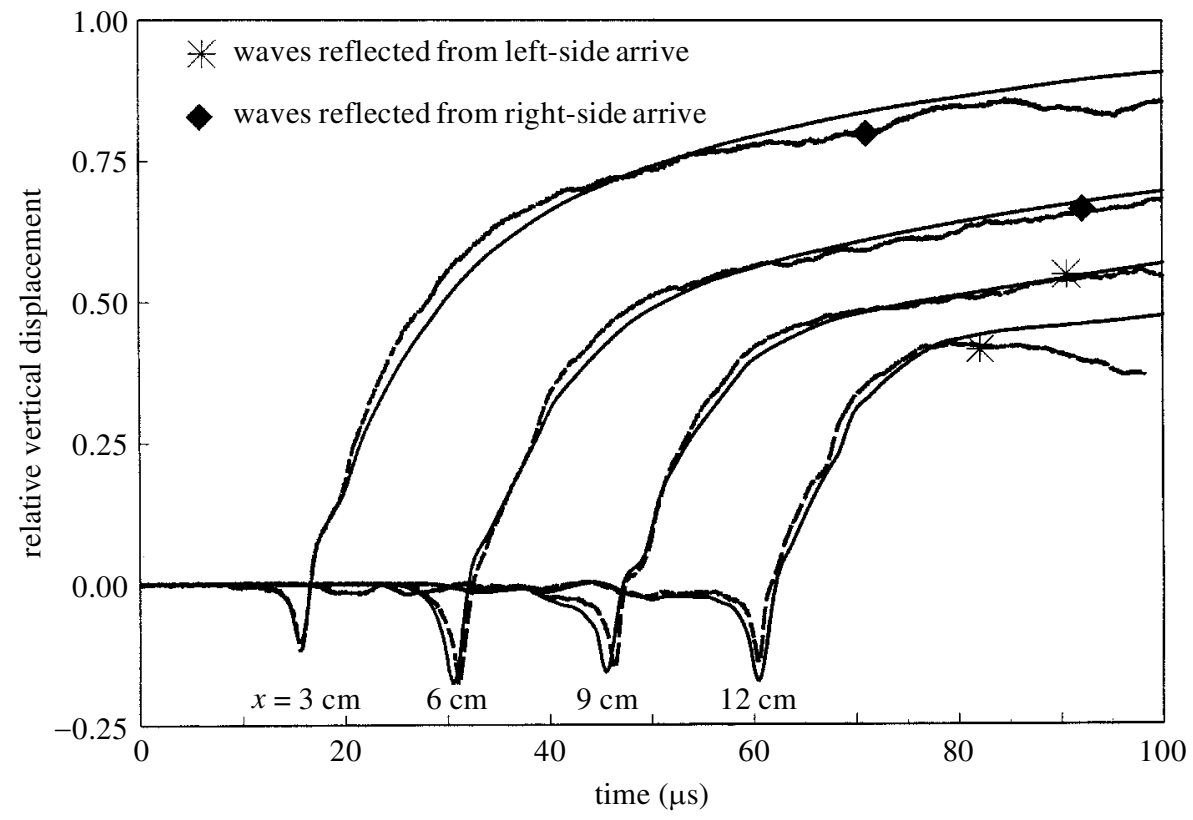

Figure 15. Comparison of measured and calculated waveforms on the free surface of specimen 3 (dashed lines for the experimental measurements, solid lines for the numerical calculations). 


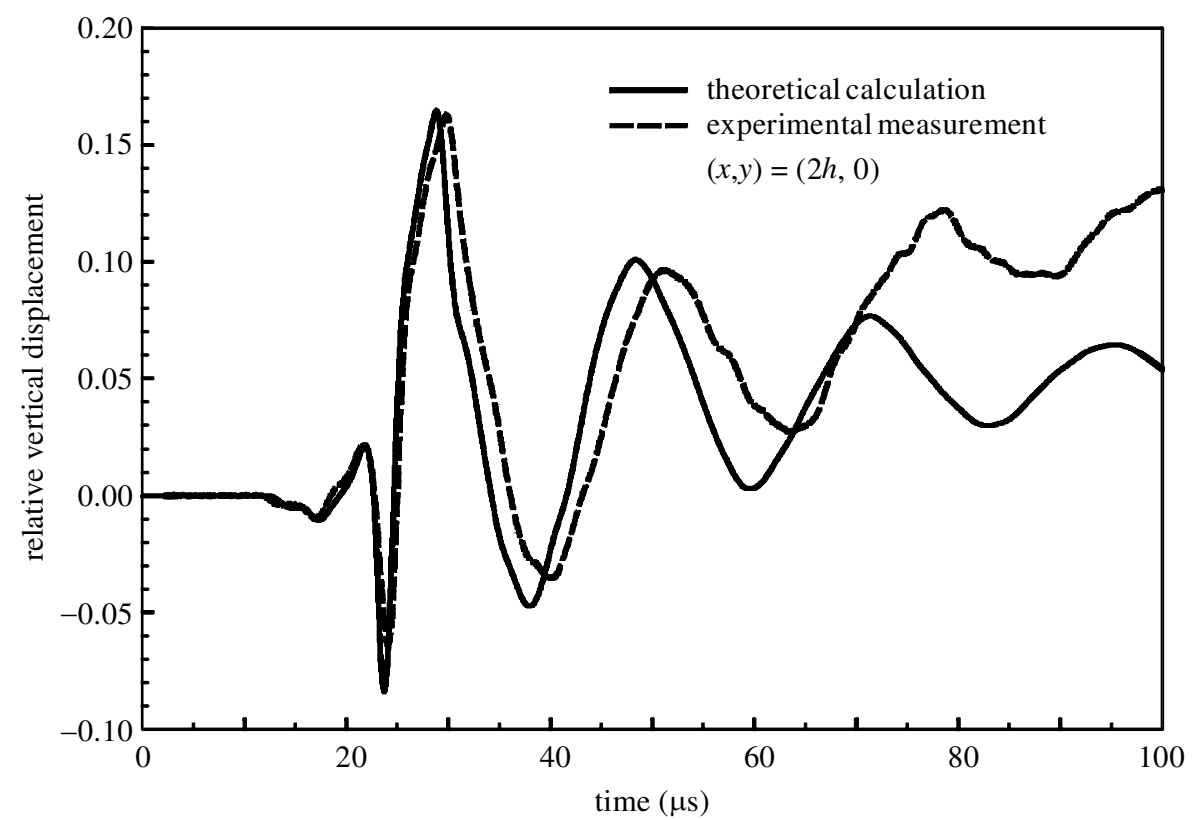

Figure 16. Comparison of measured and calculated waveforms on the free surface of specimen 4 (dashed line for the experimental measurement, solid line for the numerical calculation).

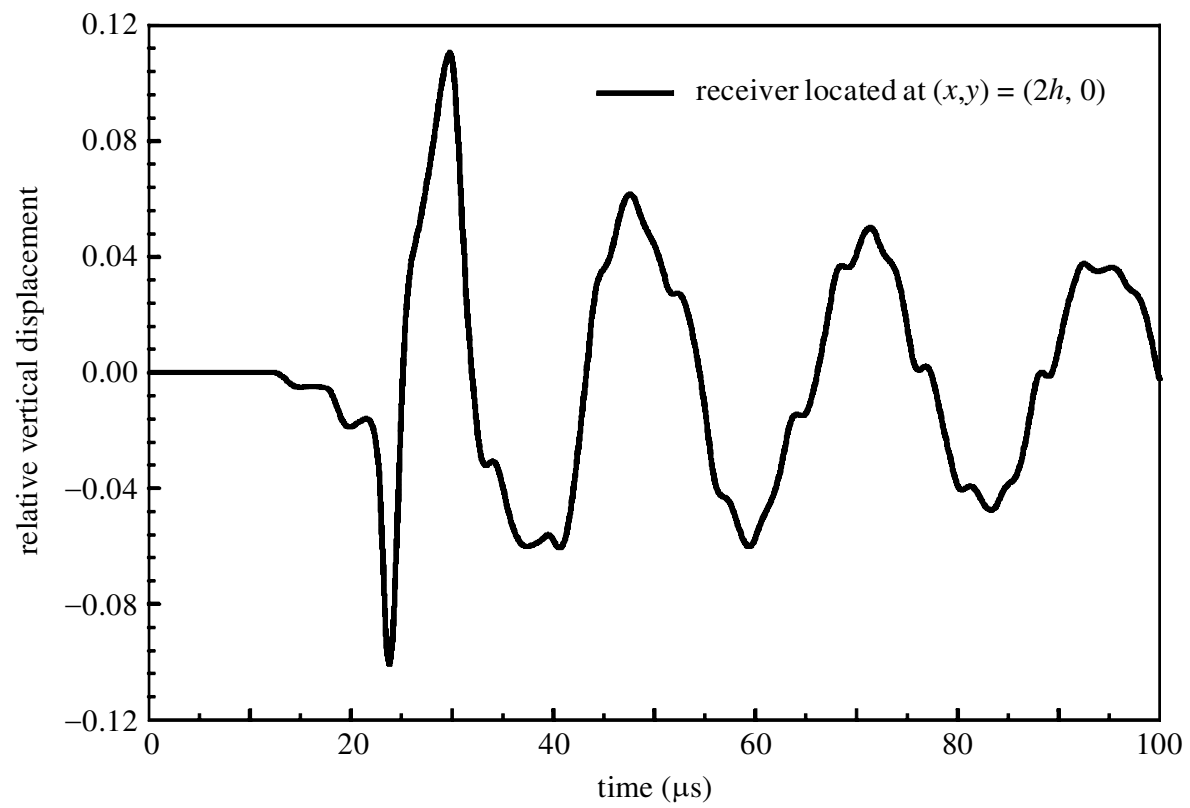

Figure 17. The vertical transient displacement on the free surface of a strip whose upper and lower surfaces are free and fixed, respectively. 
wave to the identification of perfect bonding in a layered half-space, and to the inverse evaluation of the material constants.

\section{Conclusions}

In this paper, the closed-form solution, which includes all transient waves propagating in the multi-layered medium subjected to dynamic loadings, is presented. The transient solution presented in this study is equivalent to the generalized ray solution. However, in comparison to the theory of generalized ray, the proposed solution does not need the analysis on the generalized ray paths in advance. The solution obviates the problem of degeneration that occurs in the theory of generalized ray and can be used to calculate the longer period transient responses for the multi-layered medium.

The transient responses for a layered solid overlying a half-space subjected to an in-plane dynamic loading are presented as a numerical example. It is found that Stoneley-like waves occur in the numerical calculation even when the material parameters of the problem lie outside the region in which they are predicted. The numerical calculations for displacements based on the analytic results were compared with experimental measurements. The good agreements obtained between the numerically calculated and experimentally measured signals imply the feasibility of the application of transient elastic waves in the non-destructive evaluation of material properties.

The authors gratefully acknowledge the financial support of the research by the National Science Council (Republic of China) under grant NSC 87-2212-E-002-035.

\section{References}

Abramovici, F. \& Alterman, Z. 1965 Computations pertaining to the problem of propagation of a seismic pulse in a layered solid. Meth. Comp. Phys. 4, 349-379.

Achenbach, J. D. 1973 Wave propagation in elastic solids. Amsterdam: North-Holland.

Alterman, Z. \& Karal Jr, F. C. 1968 Propagation of elastic waves in layered media by finite difference methods. Bull. Seism. Soc. Am. 58, 367-398.

Breckenridge, F. R., Tschiegg, C. E. \& Greenspon, M. 1975 Acoustic emission: some applications of Lamb's problem. J. Acoust. Soc. Am. 57, 625-631.

Cagniard, L. 1939 Reflexion et refraction des ondes seismiques progressives. Paris: GauthiersVillars. (Transl. Flinn, E. A. \& Dix, C. H. 1962 Reflection and refraction of progressive seismic waves. McGraw-Hill.)

Ceranoglu, A. N. \& Pao, Y. H. 1981 Propagation of elastic pulses and acoustic emission in a plate. ASME J. Appl. Mech. 48, 125-147.

Gilbert, F. \& Laster, S. J. 1962 Excitation and propagation of pulses on an interface. Bull. Seism. Soc. Am. 52, 299-319.

Hsu, N. N., Simmons, J. A. \& Hardy, S. C. 1977 An approach to acoustic emission signal analysis - theory and experiment. Mat. Eval. 35, 100-106.

Ma, C. C. \& Lee, G. S. 1999 Theoretical analysis, numerical calculation and experimental measurement of transient elastic waves in strips subjected to dynamic loadings. Int. J. Solid Struct. 36, 3541-3564.

Müller, G. 1968a Theoretical seismograms for some types of point-sources in layered media. I. Theory. Z. Geophys. B 34, 15-35.

Müller, G. $1968 b$ Theoretical seismograms for some types of point-sources in layered media. II. Numerical calculations. Z. Geophys. B 34, 147-162. 
Müller, G. 1969 Theoretical seismograms for some types of point-sources in layered media. III. Single force and dipole sources of arbitrary orientation. Z. Geophys. B 35, 347-371.

Pao, Y. H. 1978 Theory of acoustic emission. Elastic waves and nondestructive testing of materials (ed. Y. H. Pao), vol. 29, pp. 107-128. New York: ASME.

Pao, Y. H. \& Gajewski, R. 1977 The generalized ray theory and transient response of layered elastic solids. In Physical acoustics (ed. W. P. Mason), vol. 13, ch. 6. Academic.

Pekeris, C. L., Alterman, Z., Abramovici, F. \& Jarosh, H. 1965 Propagation of a compressional pulse in a layered solid. Rev. Geophys. 3, 25-47.

Proctor, T. M., Breckenridge, F. R. \& Pao, Y. H. 1983 Transient waves in an elastic plate: theory and experiment compared. J. Acoust. Soc. Am. 74, 1905-1907.

Sachse, W. \& Hsu, N. N. 1979 Ultrasonic transducers for materials testing and their characterization. In Physical acoustics (ed. W. P. Mason \& R. N. Thurston), vol. 14, ch. 4, pp. 277-406. Academic.

Sherwood, J. W. C. 1958 Elastic wave propagation in a semi-infinite solid medium. Proc. Phys. Soc. 71, 207-219. 